\title{
Temperature-Responsive Supramolecular Hydrogels by Ternary Complex Formation with Subsequent Photo- Crosslinking to Alter Network Dynamics
}

\author{
Lei Zou, Bo Su, Christopher J. Addonizio, Irawan Pramudya, Matthew J. Webber* \\ University of Notre Dame, Department of Chemical \& Biomolecular Engineering, Notre Dame, IN USA \\ *-
}

\section{Supporting Data and Characterization}

Figure S1- Detailed schematic of synthesis

Figure S2- Temperature-dependent ${ }^{1} \mathrm{H}-\mathrm{NMR}$ of F127-BM:CB[8] prior to UV

Figure S3- Variable temperature rheology of F127-BM without CB[8]

Figure S4- Strain sweep data of F127-BM:CB[8] hydrogels prior to UV

Figure S5- Aqueous size-exclusion chromatography before and after UV

Figure S6- UV/Vis spectroscopic quantification for reduced equivalents of $\mathrm{CB}[8]$

Figure S7- Temperature-dependent ${ }^{1} \mathrm{H}-\mathrm{NMR}$ of F127-BM:CB[8] following UV

Figure S8- Comparative rheology data before and after UV treatment

Figure S9- Strain sweep data of F127-BM:CB[8] hydrogels following UV 

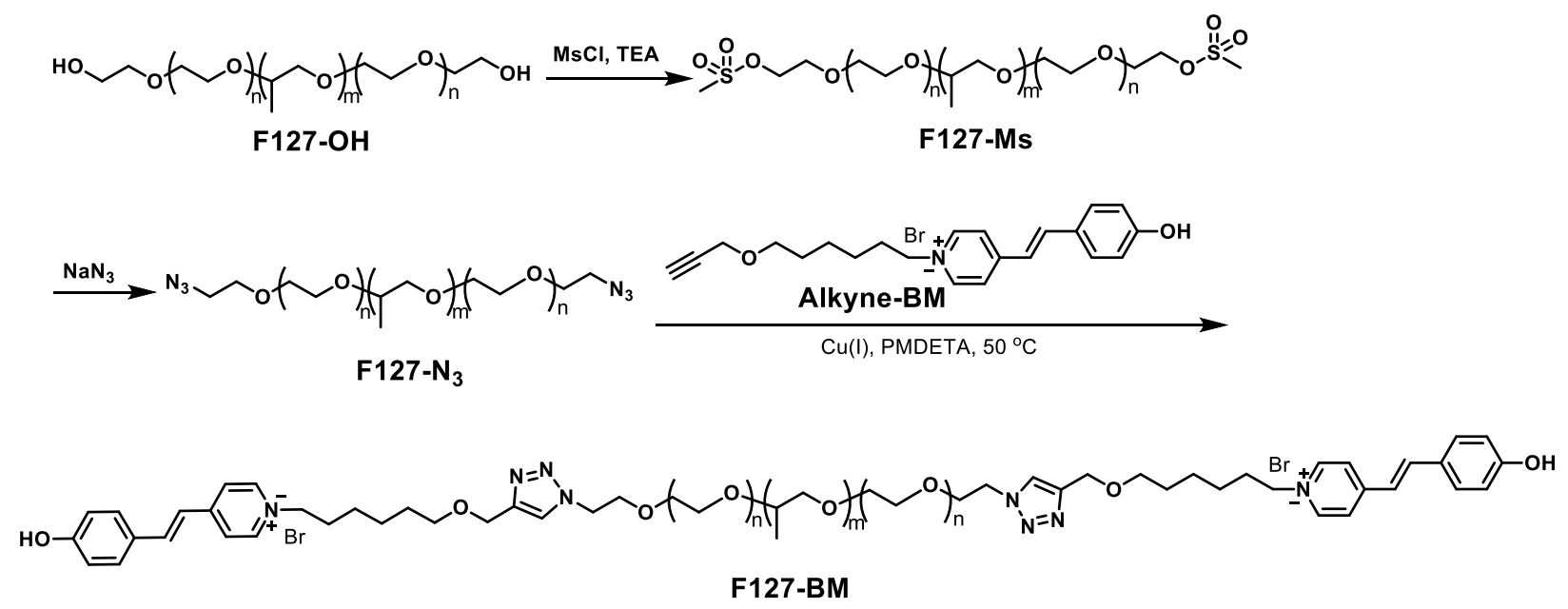

Figure S1: Detailed scheme of the reaction to append BM guests on F127 macromers.
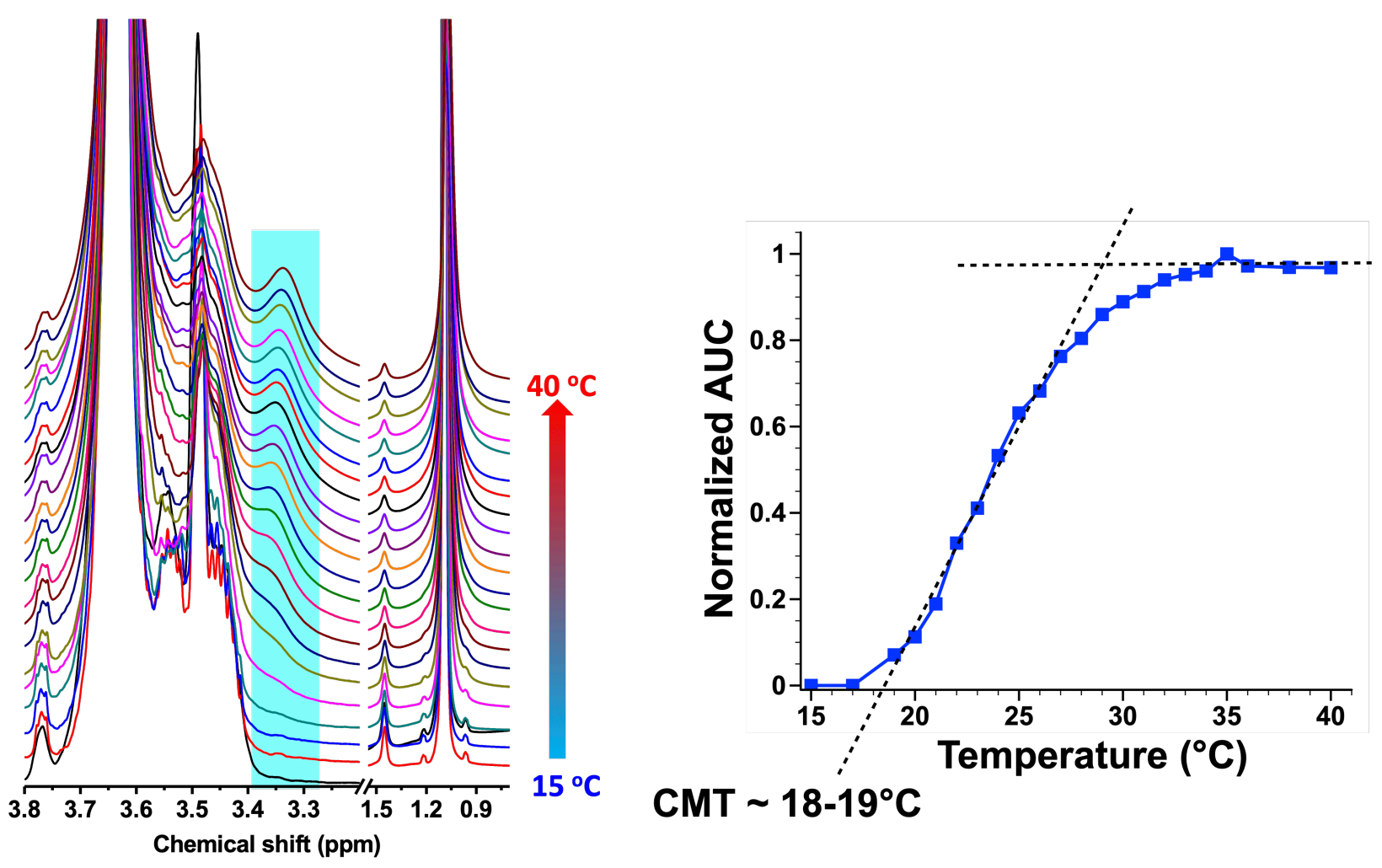

Figure S2: Temperature-dependent ${ }^{1} \mathrm{H}-\mathrm{NMR}$ (left) of F127-BM and one equivalent of CB[8] (1 $\mathrm{CB}[8]$ per $2 \mathrm{BM}$ guests), with the area under the curve in the blue-shaded region integrated to estimate the critical micelle concentration (right). 


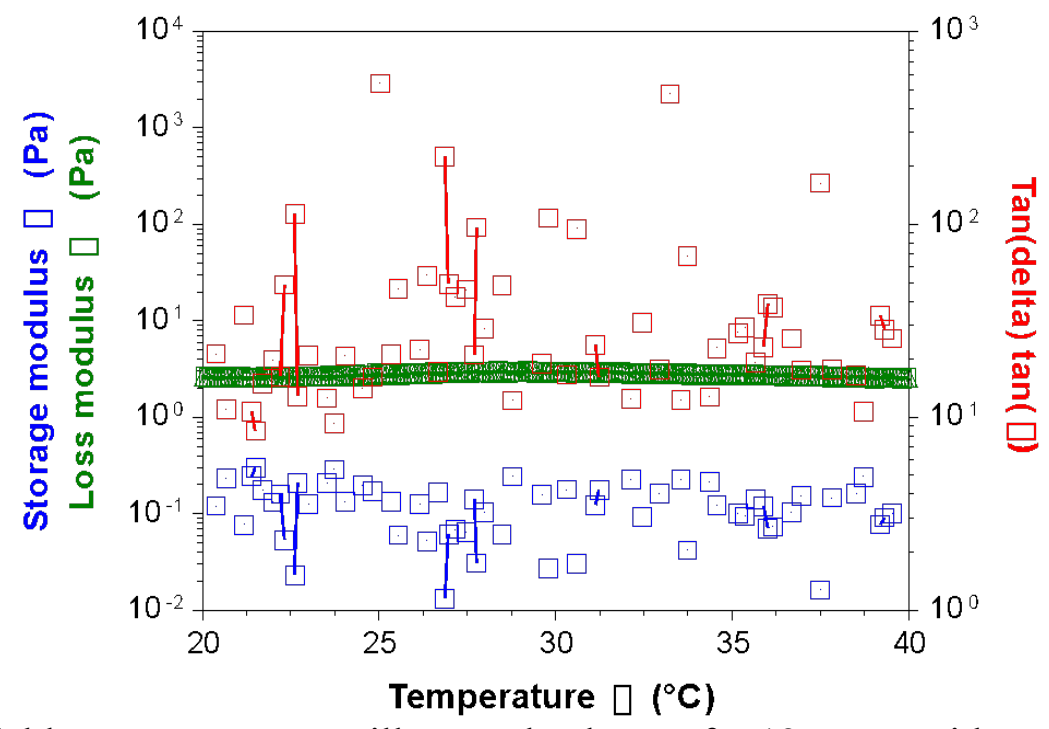

Figure S3: Variable temperature oscillatory rheology of F127-BM without CB[8], with no evidence of gelation over the full temperature range.

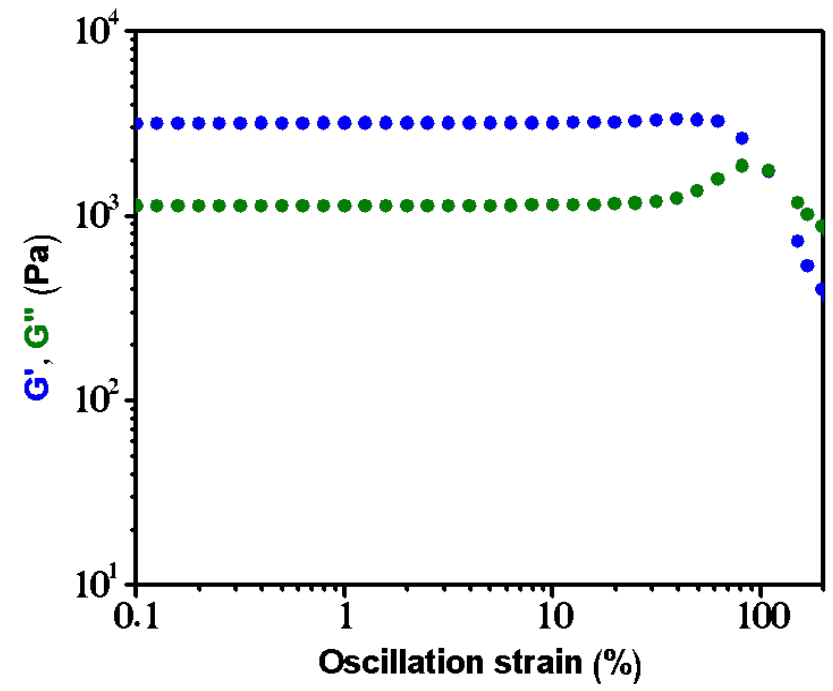

Figure S4: Strain sweep measurements of the gel formed by mixing F127-BM with 1 eq. CB[8] at $37{ }^{\circ} \mathrm{C}$ from $0.1 \%$ to $200 \%$ at $100 \mathrm{rad} / \mathrm{s}$ frequency to demonstrate the linear viscoelastic region. 


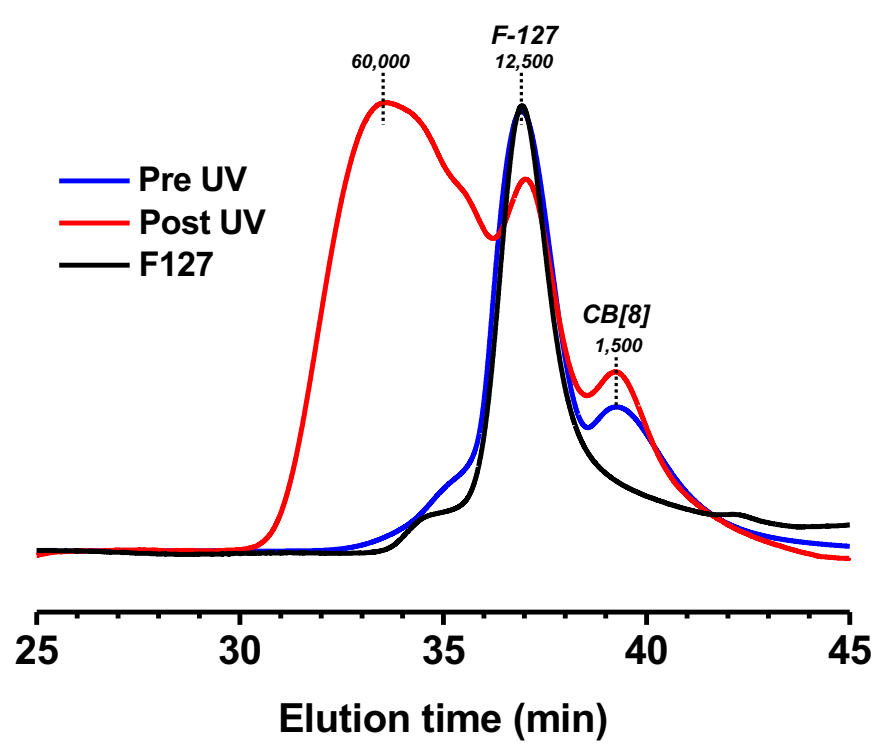

Figure S5: Size-exclusion chromatography of F127-BM with and without CB[8], as well as the sample with $\mathrm{CB}[8]$ following photodimerization. Masses for each peak were estimated on the basis of fitting to a PEG/PEO standard curve.

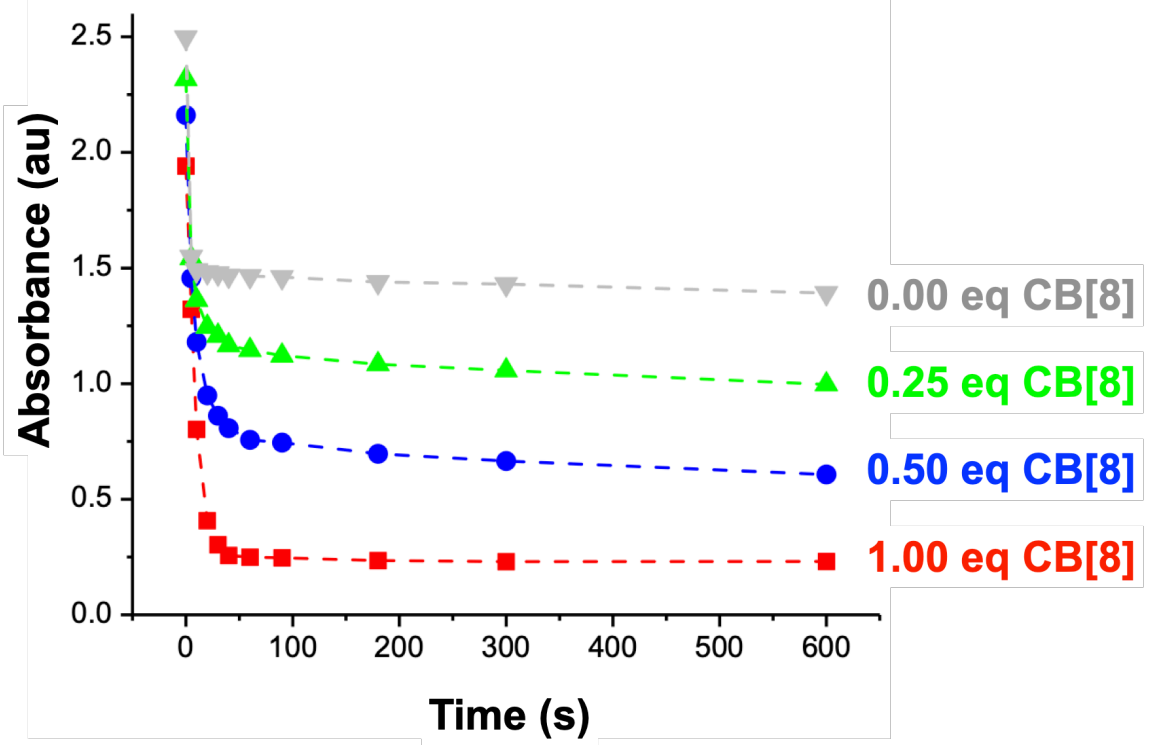

Figure S6: Peak intensity from UV/Vis spectroscopy quantifying the 378-nm absorbance signature serially with different irradiation times. 

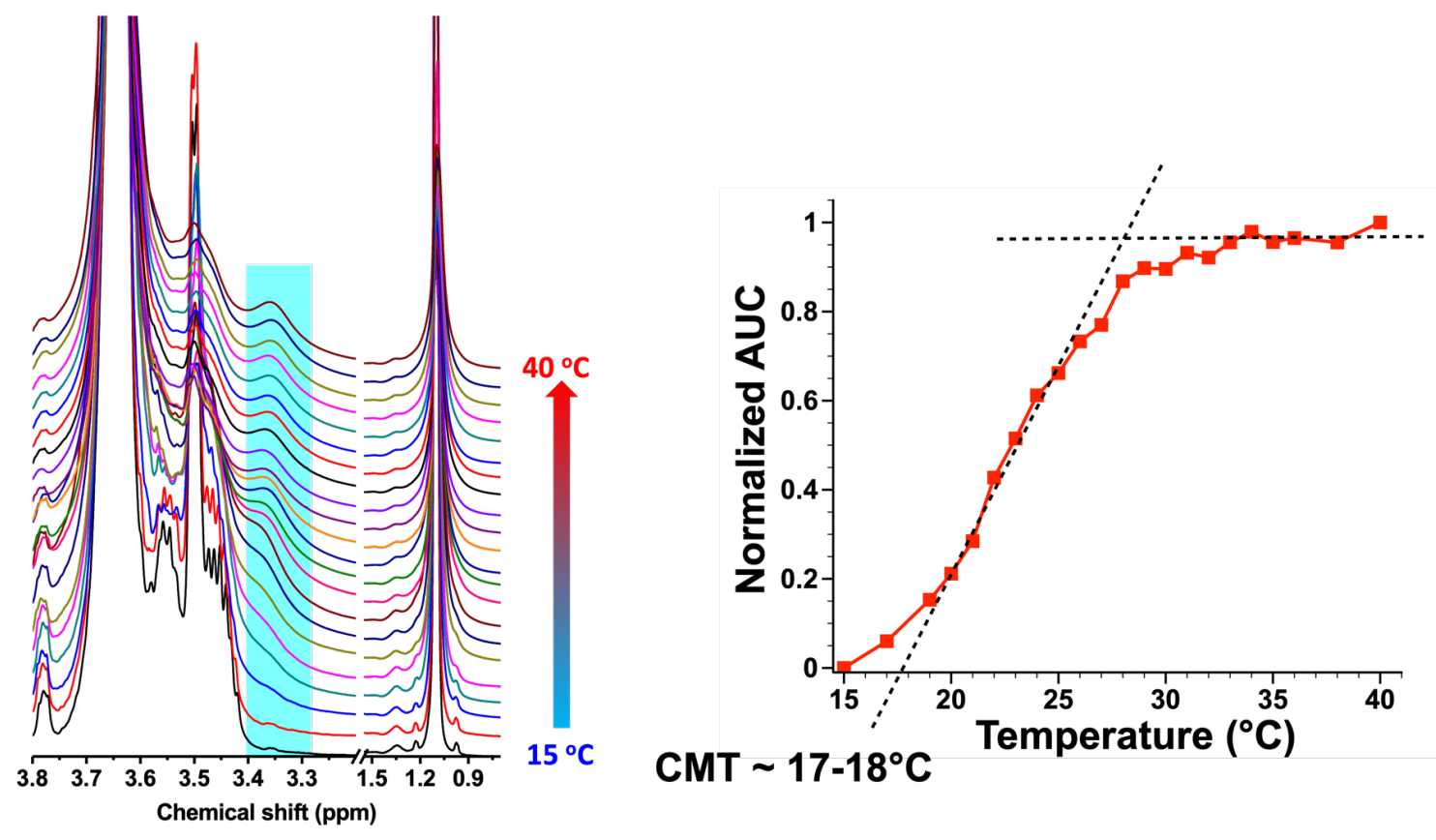

Figure S7: Temperature-dependent ${ }^{1} \mathrm{H}-\mathrm{NMR}$ (left) of F127-BM and one equivalent of CB[8] (1 $\mathrm{CB}[8]$ per $2 \mathrm{BM}$ guests) following irradiation of the polymer solution for $6 \mathrm{~h}$ with $378 \mathrm{~nm}$ light, with the area under the curve in the blue-shaded region integrated to estimate the critical micelle concentration (right). 

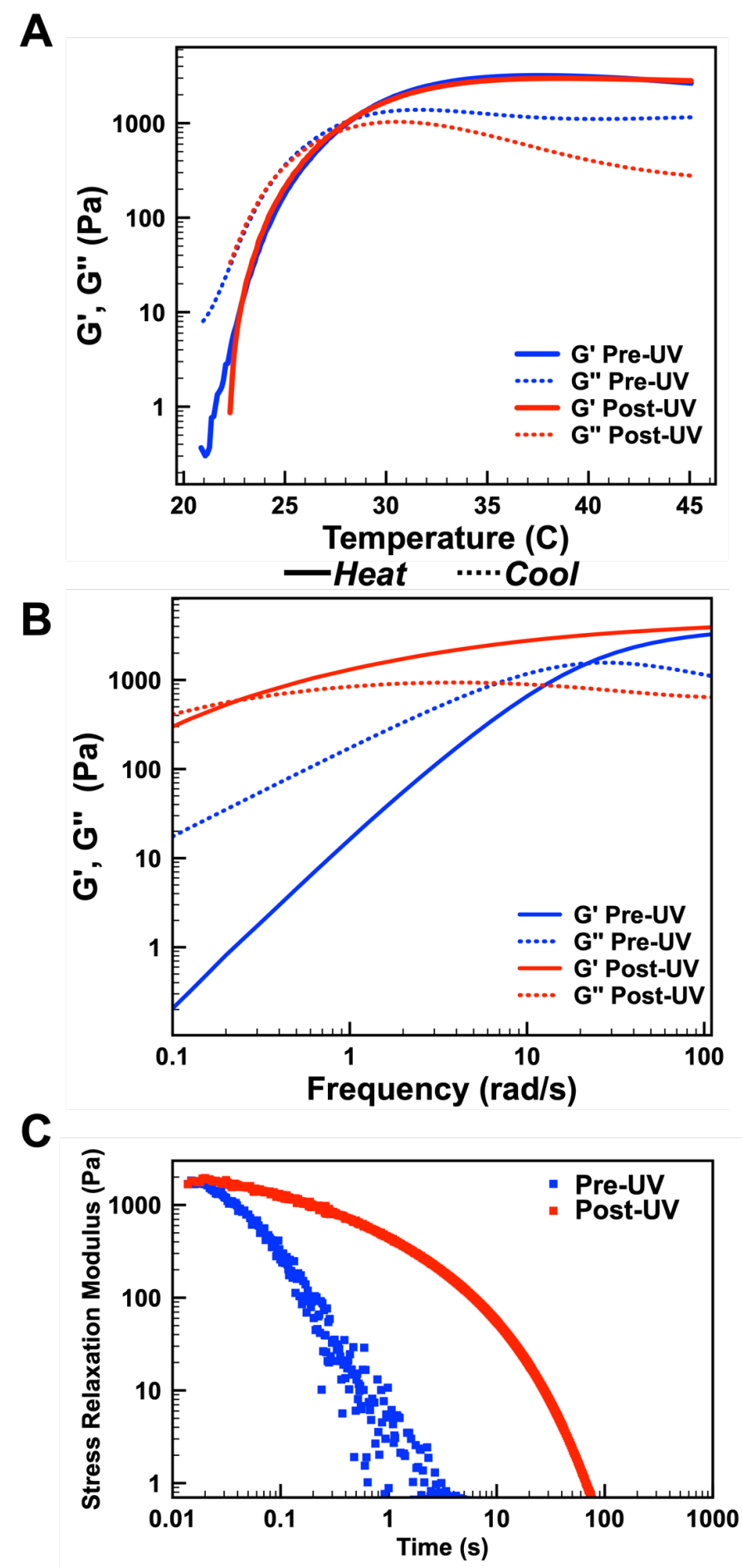

Figure S8: Comparative analysis of the rheological behavior of F127-BM:CB[8] hydrogels both before and after UV treatment to induce BM photo-dimerization, including (A) Temperaturedependent measurements, (B) Frequency Sweeps, and (C) Stress relaxation time-course studies. 


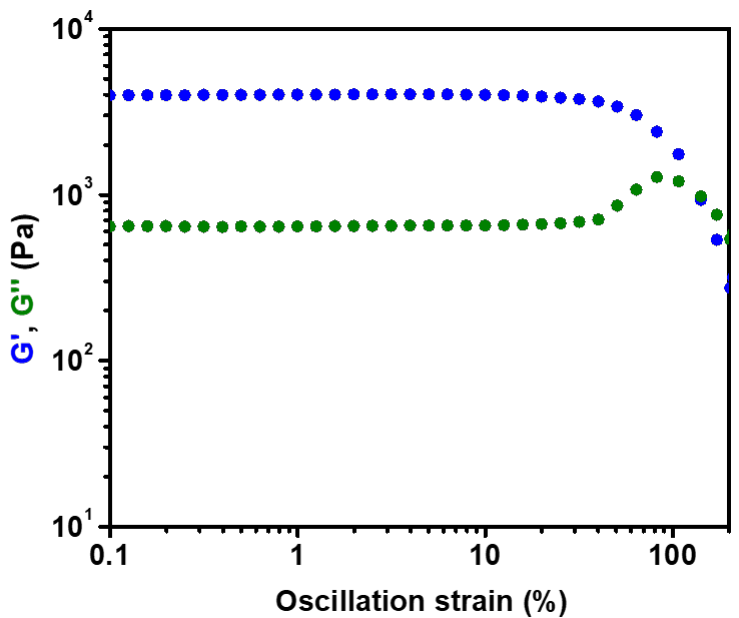

Figure S9: Strain sweep measurements of the gel formed by mixing F127-BM with 1 eq. CB[8] and irradiated for $6 \mathrm{~h}$ using $365 \mathrm{~nm}$ light, with measurements taken at $37{ }^{\circ} \mathrm{C}$ from $0.1 \%$ to $200 \%$ at $100 \mathrm{rad} / \mathrm{s}$ frequency to demonstrate the linear viscoelastic region. 